\title{
The Effect of a Sublethal Temperature Elevation on the Structure of Bacterial Communities Associated with the Coral Porites compressa
}

\author{
Jennifer L. Salerno, ${ }^{1,2}$ Dan R. Reineman, ${ }^{1,3}$ Ruth D. Gates, ${ }^{1}$ and Michael S. Rappé ${ }^{1}$ \\ ${ }^{1}$ School of Ocean and Earth Science and Technology, Hawaii Institute of Marine Biology, University of Hawaii at Manoa, \\ P.O. Box 1346, Kaneohe, HI 96744, USA \\ ${ }^{2}$ Department of Zoology, University of Hawaii at Manoa, Honolulu, HI 96822, USA \\ ${ }^{3}$ Emmett Interdisciplinary Program in Environment and Resources, Stanford University, Stanford, CA 94305, USA \\ Correspondence should be addressed to Michael S. Rappé, rappe@hawaii.edu
}

Received 15 July 2010; Accepted 30 September 2010

Academic Editor: Kim Selkoe

Copyright @ 2011 Jennifer L. Salerno et al. This is an open access article distributed under the Creative Commons Attribution License, which permits unrestricted use, distribution, and reproduction in any medium, provided the original work is properly cited.

\begin{abstract}
Evidence points to a link between environmental stressors, coral-associated bacteria, and coral disease; however, few studies have examined the details of this relationship under tightly controlled experimental conditions. To address this gap, an array of closedsystem, precision-controlled experimental aquaria were used to investigate the effects of an abrupt $1^{\circ} \mathrm{C}$ above summer ambient temperature increase on the bacterial community structure and photophysiology of Porites compressa corals. While the temperature treatment rapidly impacted the photophysiology of the coral host, it did not elicit a statistically significant shift in bacterial community structure from control, untreated corals as determined by terminal restriction fragment length polymorphism analysis of 16S rRNA genes. Two of three coral colonies harbored more closely related bacterial communities at the time of collection and, despite statistically significant shifts in bacterial community structure for both control and treatment corals during the 10-day acclimation period, maintained this relationship over the course of the experiment. The experimental design used in this study proved to be a robust, reproducible system for investigating coral microbiology in an aquarium setting.
\end{abstract}

\section{Introduction}

The worldwide degradation of coral reef ecosystems is due, in part, to the emergence of novel pathogenic diseases affecting scleractinian corals $[1,2]$, and it has been speculated that the widespread proliferation of coral diseases is linked to increasing sea surface temperatures (SSTs) [3, 4]. Many disease outbreaks correlate with temperature anomalies and seasonal warming $[3,5,6]$, and increased SSTs have also been shown to affect the virulence of coral disease pathogens [7]. For example, infection of Pocillopora damicornis by the bacterial pathogen Vibrio coralyticus increases rapidly with increased temperatures [8], and disease outbreaks often follow or co-occur with temperature-induced coral bleaching $[2,6,9]$. Discriminating between bacteria acting as causative agents of coral bleaching $[10,11]$ versus postbleaching opportunists has been ambiguous [12, 13]. However, it remains undisputed that bacteria play important roles in both maintaining and destabilizing the health of the coral holobiont, which is composed of coral host polyps, symbiotic dinoflagellates known as zooxanthellae, and a diverse assemblage of associated algae, fungi, Bacteria, Archaea, and viruses associated with the skeleton, tissues, and mucus layer of adult coral colonies [14, 15].

Our limited understanding of the resident bacterial biota associated with corals during nondiseased states hinders our ability to understand many of the processes involved in coral disease. Corals harbor diverse bacterial communities that facilitate the transformation of organic carbon $[15,16]$, acquisition and transfer of macronutrients [17, $18]$, and resistance to pathogen invasion $[19,20]$. The characterization of the diversity, function, and stability of 
these communities suggests a link between environmental stressors, coral-associated bacteria and coral disease. For example, increased temperatures, dissolved organic carbon loading, elevated nutrient concentrations, reduced $\mathrm{pH}$, and point source coastal pollution have all been shown to drive shifts in the composition of bacterial community in corals [21-23]. A shift towards "disease associated" bacteria has been observed in some cases [23] and coral mortality in others [21]. Shifts in coral-associated bacteria have also been shown to occur in corals infected with a known coral pathogen [24], and changes in bacterial biota are often detectable prior to disease symptoms becoming visible. Coral associated bacterial communities are responsive to infection by disease agents and environmental stressors. As such, the structure of the coral-associated bacterial community could potentially serve as a biological indicator of coral health.

The goals of this study were to (1) examine the feasibility and potential effects of using a closed-system, precisioncontrolled experimental aquarium system to study the structure and dynamics of microbial communities associated with corals and (2) assess the effect of a sublethal, abrupt change in temperature on coral-associated microbial community structure in the context of the photophysiological health of the coral. Shifts in the bacterial communities associated with the Hawaiian reef building coral Porites compressa were assessed in coral fragments exposed to an abrupt $1^{\circ} \mathrm{C}$ increase in seawater temperature above ambient summer levels following a $10 \mathrm{~d}$ acclimation at ambient temperature. Terminal-restriction fragment length polymorphism (T-RFLP) analysis, a bacterial community fingerprinting technique based on the 16S rRNA gene, allowed for highthroughput examinations of seawater and coral-associated bacterial community structure throughout the course of the experiment. The finger coral Porites compressa was targeted because it is endemic to Hawaii and ecologically important in the region. The photophysiological response of $P$. compressa to the temperature increase was assessed using Pulse Amplitude Modulated (PAM) fluorometry [2527], and experimental aquaria allowed for the precise control of environmental variables (such as temperature) and replication of treatments. The results show that the aquaria provided a robust platform for controlled and replicated alterations of environmental conditions that are of a relevant scale to fluctuations either experienced or anticipated to be experienced by corals in the near future, and that the thermal stress applied in this study rapidly impacted the photophysiology of the coral host. In contrast, no change in the structure of coral-associated bacterial communities was detected with the combination of temperature and duration of treatment used in this study.

\section{Methods}

2.1. Sample Collection and Experimental Design. On August 4, 2007, approximately 45 branch tips of Porites compressa (Dana 1846) 3 to $5 \mathrm{~cm}$ in length and 1 to $1.5 \mathrm{~cm}$ in diameter were removed from each of three coral colonies judged as nondiseased by gross visual assessment in central Kaneohe Bay off of the island of Oahu, Hawaii, in the

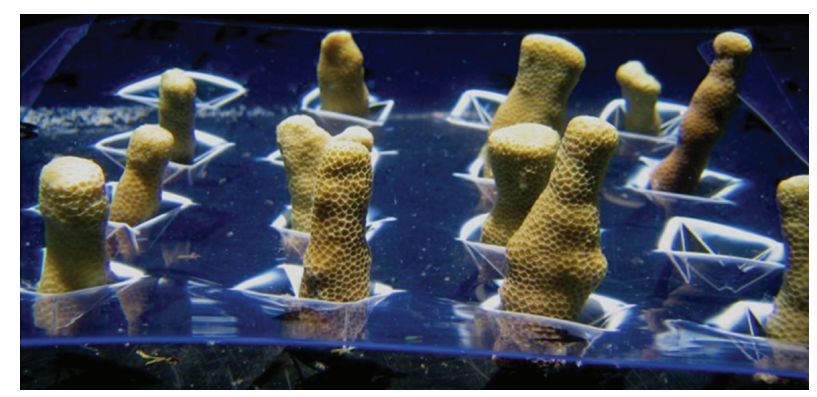

Figure 1: Photograph of $P$. compressa mounted in a vinyl sheet and held in a seawater holding tank prior to being placed into the experimental aquaria.

Pacific Ocean. Source colonies were sampled at a depth of approximately $4 \mathrm{~m}$ and were located within $10 \mathrm{~m}$ of each other. Three fragments from each colony were immediately frozen in liquid nitrogen for analysis of bacterial community structure associated with the source colonies. The remaining fragments were immediately placed in containers with aerated seawater and transported back to the Hawaii Institute of Marine Biology (HIMB). In addition to the coral sampling, water samples were collected to characterize the bacterial communities in the planktonic environment surrounding the corals. Approximately $1 \mathrm{~L}$ of seawater was collected $\sim 1.5 \mathrm{~m}$ above the reef, filtered through $25 \mathrm{~mm}$ diameter, $0.2 \mu \mathrm{m}$ pore-sized polyethersulfone membrane filters (Supor-200; Pall Corp., East Hills, NY), and frozen for DNA analysis in $250 \mu \mathrm{L}$ of DNA lysis buffer $(20 \mathrm{mM}$ Tris- $\mathrm{HCl}$ pH 8.0, 2 mM EDTA pH 8.0, 1.2\% v/v Triton X100) [28].

Upon return to the laboratory at the HIMB, coral fragments were rinsed with $1 \mu \mathrm{m}$-filtered seawater and placed in a holding tank of $1 \mu \mathrm{m}$-filtered seawater maintained at the ambient temperature of $27^{\circ} \mathrm{C}$. Four fragments from each colony were mounted randomly in each of eight $0.76 \mathrm{~mm}$ thick clear vinyl sheets (Figure 1). A sheet was placed in each of eight independently controlled $60 \mathrm{~L}$ experimental aquaria. An additional 2-4 extra fragments per colony were also mounted in sheets as backups for fragment mortality. To minimize exposure to disturbance and handling upon removal from the reef, coral samples were collected between 0900 and 0945 and mounted in their final position in the aquaria by 1400 the same day.

All aquaria were filled with $1 \mu \mathrm{m}$-filtered, UV-treated seawater, which recirculated through the individual tanks for the duration of the experiment. Each tank was equipped with a full spectrum light (white and UV) on a 12:12 hr light:dark cycle, a protein skimmer, and a submersible pump flowing at $20 \mathrm{gal} \mathrm{min}^{-1}$. The volume of seawater in each tank was monitored daily and salinities kept constant by the addition of sterile, milli-Q freshwater. Temperature and light levels in the tanks were monitored with Onset Computer HOBO temperature and light pendant loggers (UA-002-64 Onset Computer Corporation, Bourne, MA). The loggers were fixed horizontally (light meter facing up) to the vinyl sheets containing coral fragments with cable ties, and the sheets supporting the mounted corals were fixed to the bottom of 
the tank with suction cups. The seawater temperature was maintained at the ambient temperature of $27^{\circ} \mathrm{C}$ for $10 \mathrm{~d}$ to allow corals to acclimate.

After the acclimation period, four tanks were randomly assigned as controls (tanks 1, 8, 9, and 13) and four as treatment (tanks $3,4,15$, and 16). At 1300 on that day, seawater and coral bacterial communities were sampled, and the seawater temperature in the treatment tanks abruptly increased to $28^{\circ} \mathrm{C}\left(+1^{\circ} \mathrm{C}\right.$ over $)$ in treatment tanks. Corals were subsequently sampled from the aquaria at the same time of day (i.e., initiated at 1300 and completed by 1430) after 2, 4, and $6 \mathrm{~d}$ of incubation and aquarium seawater after 2 and $7 \mathrm{~d}$. Coral sampling consisted of removing one randomly selected coral fragment per colony per tank and freezing it in liquid nitrogen. Seawater $(250 \mathrm{~mL})$ was removed from each tank using acid-washed polycarbonate bottles and subsequently filtered through $25 \mathrm{~mm}$ diameter Supor-200 membranes (Pall Corp.). Filters were placed in $200 \mu \mathrm{L}$ of DNA lysis buffer and stored at $-80^{\circ} \mathrm{C}$ until further processed.

2.2. Pulse Amplitude-Modulated (PAM) Fluorometry. Pulse amplitude-modulated fluorometry was performed by taking one measurement per fragment on the fragment tip with a Walz Diving-B PAM Fluorometer (Heinz Walz GmbH, Germany) with an 8 -mm fiber optic cable. The measurement tip of the PAM cable was sheathed in opaque tubing to prevent light pollution of the other fragments during measurements and to create a standard distance between the sample and the measuring tip. Sampling was performed in the evening after approximately $1 \mathrm{~h}$ of dark adaptation. The resulting Maximum Quantum Yield (MQY) data was analyzed with Minitab 15.0 statistical analysis software (Minitab Inc, State College, PA).

2.3. T-RFLP of Bacterial SSU rRNA Genes. Individual coral fragments were thawed, and a flame-sterilized stainless steel core borer was used to remove three random subsamples. Each subsample consisted of a 6-mm diameter, 6-mm deep core that included the coral tissue, overlying mucus layer, and underlying skeleton. The three subsamples were placed into one sterile bag containing $2 \mathrm{~mL}$ of $0.2 \mu \mathrm{m}$-filtered 10X Tris EDTA (100 mM Tris, 10 mM EDTA) buffer solution ( $\mathrm{pH}$ 7.4) and airbrushed with an air gun and sterile pipette tip. The resultant slurry was centrifuged at 19,900 RCF for $30 \mathrm{~min}$ at $4{ }^{\circ} \mathrm{C}$. The supernatant was subsequently removed from the sample and the remaining sample pellet was frozen at $-80^{\circ} \mathrm{C}$ until processed further.

After thawing, genomic DNA was extracted from the coral tissue pellets and seawater filters using the PowerSoil DNA Isolation Kit (MoBio Laboratories Inc., Carlsbad, CA) according to the manufacturer's protocol. Genomic DNA was eluted in sterile, $0.1 \mu \mathrm{m}$-filtered water and stored frozen at $-20^{\circ} \mathrm{C}$. Total genomic DNA yield was assessed on a SpectraMax M2 plate reader (Molecular Device Corp., Sunnyvale, CA, USA) using the Quant-iT PicoGreen dsDNA Assay Kit (Invitrogen Corp., Carlsbad, CA, USA), prepared according to manufacturer's protocol.
For terminal restriction fragment length polymorphism (T-RFLP) analysis [29], the general bacterial primers 27FB-FAM (5'-FAM-AGRGTTYGATYMTGGCTCAG- $\left.3^{\prime}\right)$ and 1492R ( $5^{\prime}$-GGYTACCTTGTTACGACTT-3') were used for the amplification of small subunit ribosomal RNA (16S rRNA) genes from each sample via the polymerase chain reaction (PCR). The MasterTaq System (Eppendorf AG, Hamburg, Germany) was used for all PCR reactions, which were composed of the following (final concentrations): $1 \mathrm{X}$ MasterTaq reaction buffer, $2.25 \mathrm{mM} \mathrm{Mg}^{2+}, 0.5 \mathrm{X}$ TaqMaster reaction enhancer, $0.2 \mathrm{mM}$ each of the forward and reverse primers, $0.2 \mathrm{mM}$ of each dNTP (Promega, Madison, WI), approximately 160 to $280 \mathrm{ng}$ of genomic DNA template, 2.5 units of MasterTaq DNA polymerase, and sterile water to a final reaction volume of $50 \mu \mathrm{l}$. A MyCycler thermal cycler (Bio-Rad Laboratories, Hercules, CA, USA) and touchdown protocol were used, which, after a $3 \mathrm{~min}$ incubation at $95^{\circ} \mathrm{C}$, consisted of 30 cycles of $30 \mathrm{sec}$ at $95^{\circ} \mathrm{C}, 1 \mathrm{~min}$ at $65^{\circ} \mathrm{C}$ (decreasing by $0.5^{\circ} \mathrm{C}$ per cycle), and $2 \mathrm{~min}$ at $72^{\circ} \mathrm{C}$. This was followed by 10 cycles of $30 \mathrm{sec}$ at $95^{\circ} \mathrm{C}, 1 \mathrm{~min}$ at $50^{\circ} \mathrm{C}$, and $2 \mathrm{~min}$ at $72^{\circ} \mathrm{C}$, and 1 cycle of $30 \mathrm{sec}$ at $95^{\circ} \mathrm{C}, 1 \mathrm{~min}$ at $50^{\circ} \mathrm{C}$, and $20 \mathrm{~min}$ at $72^{\circ} \mathrm{C}$.

The fluorescently labeled amplicons were purified using the QIAquick PCR purification kit (Qiagen Inc.) following the manufacturer's instructions. Approximately $100 \mathrm{ng}$ of each purified amplicon was subsequently digested in a $10 \mu \mathrm{L}$ reaction containing 5 units of HaeIII restriction endonuclease (Promega, Madison, WI) at $37^{\circ} \mathrm{C}$ for 6 hours. After purification via gel filtration chromatography with Sephadex G-50 (Amersham Biosciences, Sweden), the restricted samples were adjusted to a final concentration of $35 \mathrm{ng} \mu \mathrm{L}^{-1}$ and separated via capillary electrophoresis on an automated ABI 3100 Genetic Analyzer (Applied Biosystems, Foster City, CA). GeneMapper software (Applied Biosystems) was used to estimate the size and relative abundance of the resulting terminal restriction fragments (T-RFs), which were defined as fragments between 42 and 613 base pairs (bp) in length. Fragment lengths were rounded to the nearest integer value, aligned, and manually checked for possible errors in peak determination due to such factors as instrument variability, and so forth. The threshold below which peaks were excluded was determined via the variable percentage threshold method as described in Osborne et al. [30].

2.4. Statistical Analysis. After square root transformation of the T-RFLP data matrix, all subsequent statistical analyses were carried out in PRIMER 6 Version 6.1.13 and PERMANOVA + Version 1.0.3 (PRIMER-E Ltd., Plymouth, UK) [31-33].

A resemblance matrix was constructed based on BrayCurtis similarity. Hierarchical clustering analysis (CLUSTER) of the resemblance matrix was used to construct similarity dendrograms, and a similarity profile permutation test (SIMPROF) was used to identify significant "natural groupings" of samples that were not structured a priori. CLUSTER analyses were based on the group average linking option and SIMPROF analyses were performed at a 5\% significance level. 
TABLE 1: PAM-derived maximum quantum yield regression values during acclimation and manipulation phases of $P$. compressa.

\begin{tabular}{lccccc}
\hline Group & Time & Slope & $R^{2}$ & $T$ statistic & $P$-value \\
\hline Control & Acclimation & -0.00168 & 0.052 & -3.61 & .000 \\
& Manipulation & -0.00392 & 0.070 & -3.26 & .001 \\
Treatment & Acclimation & -0.00159 & 0.049 & -3.54 & -7.43 \\
& Manipulation & -0.00875 & 0.273 & .000 \\
\hline
\end{tabular}

Nonmetric multidimensional scaling (NMDS) ordination of the resemblance matrix was used to provide a 2-dimensional graphical representation of similarities in bacterial community structure (based on T-RFLP peak abundances) amongst samples [34, 35]. NMDS plots can be generally interpreted as follows: points that are closer together are very similar in community composition, and points that are further apart correspond to very different communities. NMDS was chosen because it makes no assumptions about the underlying distribution of data and is therefore appropriate for analyzing complex ecological communities. In some cases, data from CLUSTER analyses were used to overlay Bray-Curtis similarity boundaries onto NMDS plots to display percent similarity amongst samples.

An analysis of similarity (ANOSIM), performed on the resemblance matrix, was used to test for differences in bacterial community structure between predefined sample groups. The ANOSIM test is analogous to a standard univariate 1-way analysis of variance (ANOVA), and group specifications were made a priori. A "similarity percentages" routine (SIMPER) was used to identify the role of individual species in contributing to group differences as well as within group closeness. SIMPER dissects average Bray-Curtis dissimilarities between all pairs of samples into percentage contributions from each species and lists them in decreasing order of contribution [31]. An $80 \%$ cutoff was employed to focus on higher-contributing variables.

A permutational multiple analysis of variance (PERMANOVA) of the resemblance matrix was used to test for differences in bacterial community structure between temperature treatments, tanks, coral colonies, and time points. PERMANOVA tests the simultaneous response of one or more variables to one or more factors in an analysis of variance (ANOVA) experimental design on the basis of a resemblance measure, using permutation methods. The routine calculates an appropriate distance-based pseudo$F$ statistic for each term in the model based on the expectations of mean squares, and $P$-values are obtained using an appropriate permutation procedure for each term [32]. PERMANOVA tests were run with the following specifications: 999 permutations, permutation of residuals under a reduced model, Type III (partial) sum of squares, Monte Carlo tests, and fixed effects sum to zero.

\section{Results}

3.1. Photophysiology of P. compressa. Initially, both control and treatment fragments of $P$. compressa exhibited very high variability in MQY, presumably due to shock associated with their collection from the field, loading into sheets, and

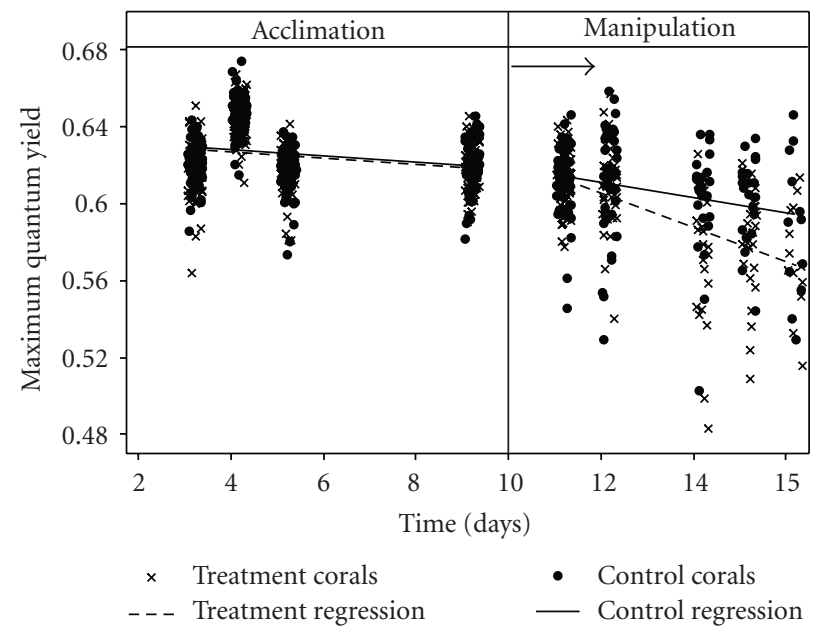

FIGURE 2: Maximum quantum yield of $P$. compressa during acclimation to experimental tanks (acclimation) and after a temperature increase of $1^{\circ} \mathrm{C}$ at Day 10 (treatment group tanks only; manipulation). Each point represents a single PAM measurement (points are shifted slightly in the $\pm \mathrm{x}$ direction in order to reduce overlap). Lines are least-squares linear regressions. Control colonies: closed circles, solid regression; treatment colonies: x's, dashed regression. Note: the treatment and control regression lines overlap during the "acclimation" phase.

positioning within the aquaria (data not shown). By Day 3, however, the MQY had stabilized and remained nearly constant throughout the acclimation period (Figure 2). Statistical analyses revealed that the MQY of the control and treatment groups did not differ significantly during this acclimation phase (two sample $t$-test: $t=0.74, \mathrm{DF}=$ 481 , and $P=.459$ ), indicating there were no tank effects. In addition, the MQY of all colonies on the first and last days of the acclimation period were also not significantly different (two sample $t$-test: $t=0.17, \mathrm{DF}=239, P=$ .867 ), indicating that the MQY stabilized during acclimation. After the initiation of the temperature manipulation on Day $10\left(+1^{\circ} \mathrm{C}\right)$, the MQY of both treatment and control samples decreased. However, linear regression of the control and treatment samples indicated a greater decline in the treatment samples over time (Table 1; Figure 2) with the slope of the control sample linear regression decreasing by a factor of 2.33 and the slope of the treatment by a factor of 5.50 .

3.2. Planktonic Bacterial Community Analysis. CLUSTER and ANOSIM analyses of all coral-associated $(n=99)$ and seawater bacterial community $(n=26)$ samples collected 


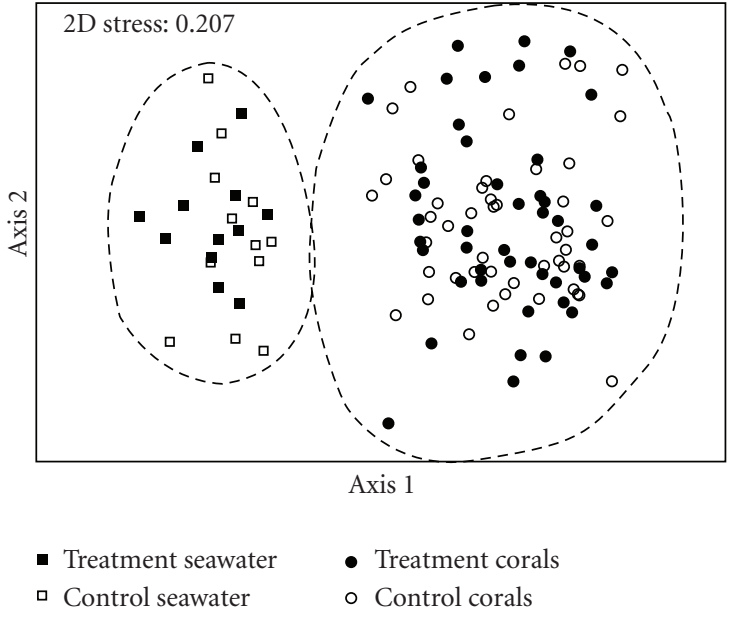

FIgURE 3: NMDS ordination of all coral $(n=99)$ and seawater ( $n=26$ ) bacterial communities sampled over the course of this study, based on a Bray-Curtis similarity matrix calculated from a square root transformation of the T-RFLP relative abundance data matrix. The dashed line indicates the $20 \%$ Bray-Curtis similarity threshold. Seawater samples are represented by squares and coral samples are represented by circles. Control and treatment samples are represented by open and closed symbols, respectively.

over the course of this study showed that the bacterial communities were significantly different between the two sample types (Global $R=0.864, P=.001$ ). An initial NMDS ordination of all coral and seawater samples overlaid with a $20 \%$ Bray-Curtis similarity boundary resolved two distinct clusters clearly demonstrating this difference (Figure 3 ).

The microbial community in seawater from Kaneohe Bay taken near the corals sampled for this experiment $(n=1)$, from the source water produced by the HIMB seawater system $(n=1)$, and from the aquaria throughout the course of the experiment $(0,2$, and 7 days; $n=24)$ were compared using CLUSTER and ANOSIM. These tests revealed a tight group of microbial communities from the seawater sample from Kaneohe Bay and from the HIMB seawater system, which was significantly different from the aquaria planktonic microbial communities, regardless of whether they originated from control or treatment tanks (Figure 4) (Global $R=0.981, P=.003$ ). Thus, the planktonic bacterial community structure within the aquaria shifted over the 10 days between the time of collection and start of the experiment. A PERMANOVA test was used to investigate the differences in seawater bacterial communities amongst different aquaria throughout the experiment. This test revealed no difference between control or treatment tanks (Pseudo-F $=0.619, P=.824$ ). However, the test did reveal differences in the bacterial communities in aquarium seawater over time (Pseudo- $\mathrm{F}=2.173, P=.003$ ) and between replicate tanks within control and treatment (Pseudo-F $=2.119, P=.001$ ). An ANOSIM test confirmed differences in aquarium seawater bacterial communities over time (Global $R=0.131, P=.019$ ), but only identified time points $0 \mathrm{~d}$ and $7 \mathrm{~d}$ as being significantly different from one another $(R=0.33, P=.003)$. Differences between

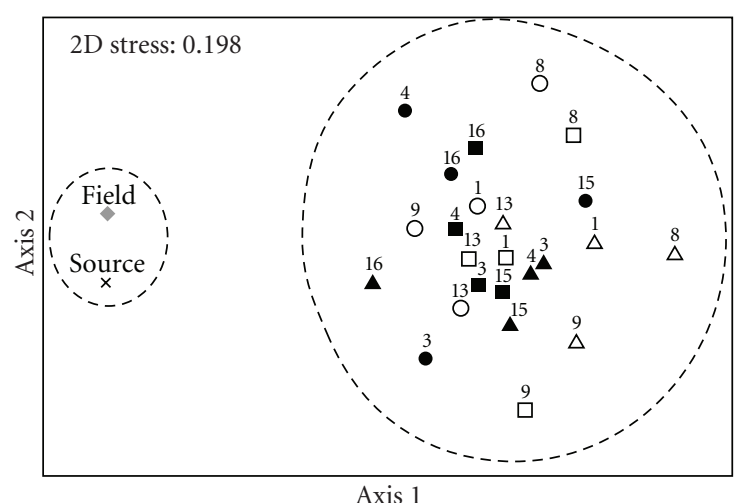

$\begin{array}{ll}\quad \text { Control } & \text { Treatment } \\ \bigcirc t=0 & \bullet t=0 \\ \square t=2 \mathrm{~d} & \square t=2 \mathrm{~d} \\ \Delta t=7 \mathrm{~d} & \Delta t=7 \mathrm{~d}\end{array}$

Figure 4: Comparison of seawater bacterial communities sampled over the course of this study $(n=26)$, based on a NMDS ordination of a Bray-Curtis similarity matrix calculated from a square root transformation of the T-RFLP relative abundance data matrix. The dashed line indicates the $20 \%$ Bray-Curtis similarity threshold. "Field" indicates the seawater bacterial community sampled from the field site in Kaneohe Bay, while "source" indicates the seawater system at HIMB used as a source for the aquaria. Control aquaria are represented by open symbols and are individually numbered $1,8,9$, and 13 , while treatment aquaria are represented by closed symbols and are individually numbered $3,4,15$, and 16 . Sampling times are indicated by circles $(t=0)$, squares $(t=2 \mathrm{~d})$, and triangles $(t=7 \mathrm{~d})$.

tanks within control or treatments were also confirmed via ANOSIM (Global $R=0.372, P=.001$ ), and pairwise comparisons revealed a range of similarities between tanks from both the control and treatment sets. For example, control tank 8 was highly distinct from the other three control tanks, with $R$ values approaching $1(8 / 9 R=0.704$; $8 / 1 R=0.852 ; 8 / 13 R=0.889)$ whereas control tank 4 and treatment tank 13 were highly similar $(R=0)$.

3.3. Coral-Associated Bacterial Community Analysis. Coral samples collected from source colonies in the field $(n=3)$ were compared to all experimental and control coral samples $(0,2,4$, and $6 \mathrm{~d}$ time points; $n=96)$ using CLUSTER and ANOSIM tests, which revealed a significant difference between source and aquaria maintained colonies (Global $R=0.688, P=.001)$. Thus, coral associated bacterial community structure appeared to shift between the time of collection and the start of the experiment $10 \mathrm{~d}$ later. A PERMANOVA test including all time points indicated that coral-associated bacterial communities did not differ between the control or treatment sets (Pseudo-F $=0.791$, $P=.807$ ) or over time (Pseudo $-F=1.257, P=.145$ ). Despite significant differences between tanks within control or experimental tank sets (Pseudo-F $=1.532, P=.003$ ), significant intercolony differences in bacterial community structure were maintained over the course of the experiment across all tanks (Pseudo- $\mathrm{F}=8.338, P=.001$ ). 
To further investigate intracolony differences, separate PERMANOVA tests were performed for each colony. This confirmed that bacteria associated with colonies 2 and 3 were not significantly affected by the treatment (Pseudo-F = 0.900, $P=.502$; Pseudo-F $=1.068, P=.377)$, time (Pseudo-F $=1.433, P=.062$; Pseudo-F $=0.842, P=$ .754 ), or tank (Pseudo-F $=1.232, P=.110$; Pseudo- $\mathrm{F}=$ $1.009, P=.487)$. While test results for colony 1 revealed that bacterial community structure was not significantly affected by treatment (Pseudo-F $=0.846, P=.793$ ), it was significantly affected by tank (Pseudo-F $=1.305$, $P=.036$ ) and time (Pseudo-F $=1.524, P=.017)$. An ANOSIM test of colony 1 data supported these observations (Global $R=0.097, P=.028$ ), with significant differences between time points 0 and $4 \mathrm{~d}(R=0.218, P=.015)$ and between 2 and $6 \mathrm{~d}(R=0.209, P=.043)$, but not between 0 and $2 \mathrm{~d}(R=0.111, P=.136), 2$ and $4 \mathrm{~d}(R=$ $-0.049, P=.716), 4$ and $6 \mathrm{~d}(R=0.027, P=.352)$, or 0 and $6 \mathrm{~d}(R=0.106, P=.095)$. SIMPER analysis indicated that the terminal restriction fragment of 303 base pairs in length (T-RF 303) was the major contributor to observed differences between times, accounting for $4.85 \%$ of the average dissimilarity between time 0 and $4 \mathrm{~d}$ groups and $5.37 \%$ of average dissimilarity between 2 and $6 \mathrm{~d}$ groups. The relative abundance of T-RF $303 \mathrm{bp}$ increased during the intermediate time points in control tanks 1, 8, and 13, and treatment tank 16, thus contributing to observed differences amongst tanks.

An NMDS ordination of the data set illustrated the shift in bacterial community structure between colonies sampled in the field and those sampled during the experimental time points (Figure 5(a)). The NMDS ordination also revealed that bacterial communities associated with source colonies 2 and 3 were more similar to one another than to colony 1. In spite of the overall shift in community structure between source and experimental coral-associated microbial communities observed for all three colonies, inter-colony differences were preserved over the course of the experiment: colonies 2 and 3 remained similar, while colony 1 remained distinct (Figure 5(a)). An ANOSIM test confirmed that the three colonies were significantly different from one another during the experiment (Global $R=0.33, P=.001$ ), with pairwise comparisons highlighting that colonies 2 and 3 were more similar to one another than to colony $1(2 / 3 R=0.164$; $2 / 1 R=0.427 ; 3 / 1 R=0.437 ; P=.001$ for all tests).

SIMPER was used to identify individual T-RFs that contributed most greatly to group differences and were defined within group similarities. Overall, T-RF 303 and T-RF 264 played large roles in discerning inter-colony differences (Figures 5(b) and 5(c)). T-RF 303 contributed to $6.61 \%$ of average dissimilarity between colonies 1 and 2 and $6.48 \%$ between colonies 1 and 3. T-RF 264 contributed to $4.78 \%$ of average dissimilarity between colonies 1 and 2 , and $5.78 \%$ between colonies 1 and 3 .

\section{Discussion}

As the integrity of coral reefs decline on a global scale, the development of effective means to monitor coral health has become critical. In particular, methods that detect the onset of physiological stress in corals prior to disease or death are essential for effective management. In addition to their utility in other areas of coral health and disease, we hypothesized that fluctuations in bacterial community structure associated with corals could be used as a sensitive indicator of physiological stress by the coral host. However, most studies to date that have related coral-associated bacterial community structure with coral health have focused on distant end-members (e.g. "healthy" versus "nonhealthy" or diseased) [24, 36, 37] and have not been able to tease apart the large gradient of health states in between these widely separated extremes. Experimental aquarium systems provide an opportunity to tightly control the environmental conditions experienced by the corals (and thus their associated microbiota) and consequently allow for the investigation of small, systematic, and ecologically relevant environmental disturbances.

In general, corals live within a relatively narrow temperature margin and will bleach in response to both high and low sea surface temperature anomalies [38, 39]. Bleaching due to elevated seawater temperatures occurs frequently, and even a small increase (e.g. $1-2^{\circ} \mathrm{C}$ ) for several weeks during the summer season can induce bleaching [39, 40]. Using these observations as rationale, we exposed fragments of $P$. compressa to a one degree, sublethal temperature elevation over the ambient summer temperature at the time of sampling. This temperature increase elicited a rapid response by the coral host, resulting in a progressive decrease in MQY over the course of the six-day treatment, which we interpret to indicate a decrease in the photosynthetic efficiency of the coral. The control corals that were not subjected to the temperature increase also showed a progressive but far less dramatic decrease in MQY over time. Thus, while unidentified components of the experimental aquarium set up appeared to have a negative impact on the photosynthetic efficiency of the coral with time, the additive adverse effect of the minor increase in temperature was of significant enough magnitude to overshadow the tank effect. By contrast, after $6 \mathrm{~d}$ of experimental manipulation, no clear systematic segregation was detected between bacterial communities associated with control and treatment corals.

Our results do not imply that coral-associated bacterial communities were static: these communities changed significantly during the 10 -day acclimation period when no treatment was applied, resulting in a distinct difference between the parent "source" colonies and the experimental coral fragments. Despite this divergence from source colony community structure during the acclimation period, for the most part, the coral-associated bacterial communities did not continue to systematically change during the treatment period. We interpret this to indicate that the coral-associated microbial community structure changed in response to acclimation to the experimental aquarium system, stabilized over the course of the initial 10-day period, and subsequently did not systematically respond to the temperature elevation treatment or experimental aquarium environment. The most plausible explanation for this observation is that the temperature increase was not significant enough in magnitude and/or duration to either directly impact the 


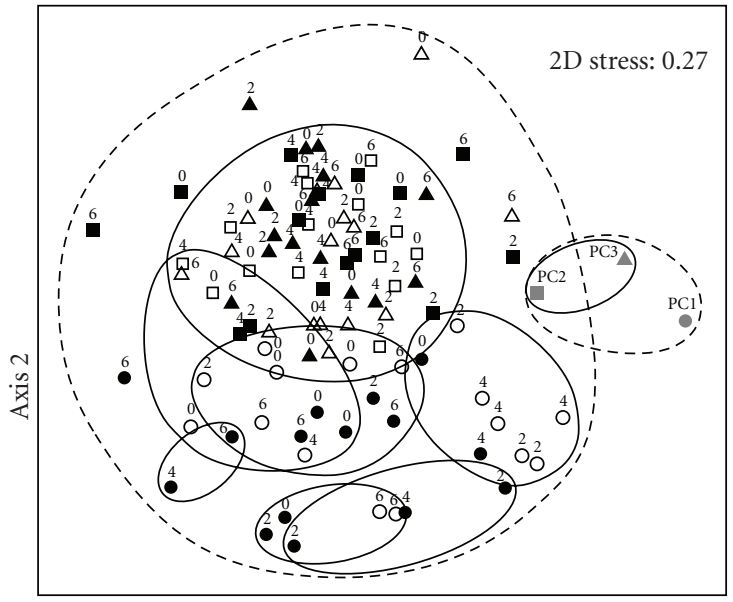

Axis 1

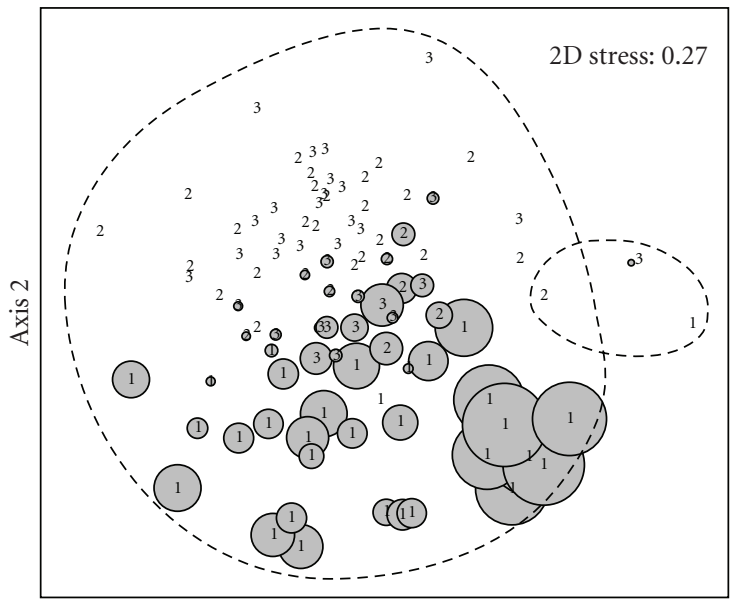

Axis 1

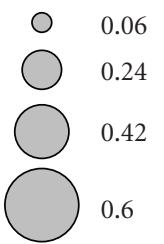

(a)

(b)

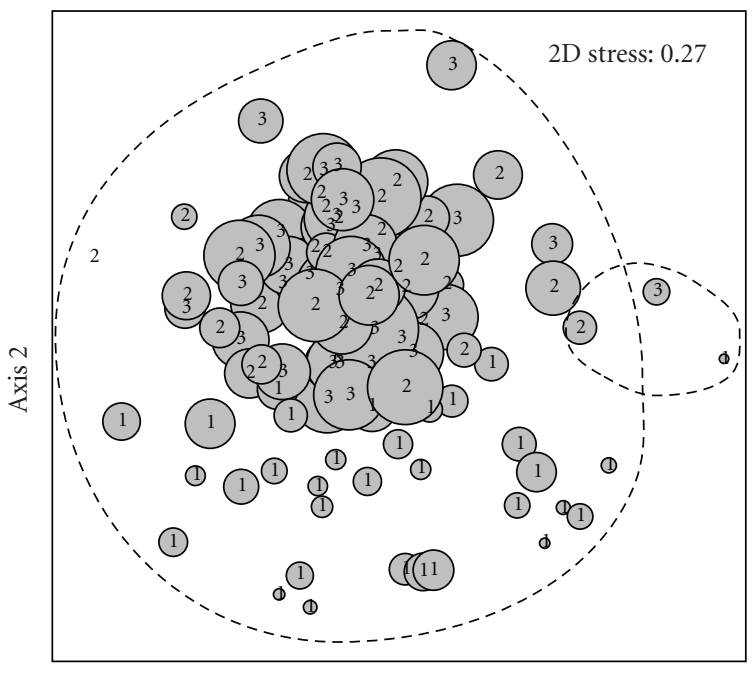

Axis 1

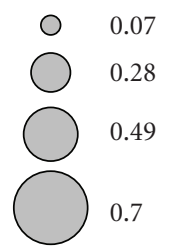

(c)

Figure 5: NMDS ordination of coral-associated bacterial communities based on Bray-Curtis similarity. Points represent coral-associated bacterial communities sampled directly from the three source colonies in Kaneohe Bay (PC1, PC2, and PC3) and from experimental tanks. (a) Sampling times are listed adjacent to points, and colonies are represented by circles (colony 1), squares (colony 2), and triangles (colony 3). Control and treatment samples are represented by open and closed symbols, respectively. In (b) and (c), colony numbers are superimposed on the relative abundance bubbles, and the diameter of each bubble is correlated to the square root transformed relative abundance of (b) T-RF 303 or (c) T-RF 264 for each sample. The solid (a) and dashed (a)-(c) lines demarcate 40\% and 25\% Bray-Curtis similarity thresholds, respectively. 
growth of the coral-associated bacterial biota or indirectly effect their growth by impacting the physiology of the coral to a degree that would impact the microbial community. In essence, PAM fluorometry appeared to be detecting changes in the photophysiology of the coral host that had no impact on the community structure of associated bacteria for the duration of our experiment. In future experiments, it will be valuable to extend the duration of the temperature treatment significantly (i.e., from days to weeks) to correlate bacterial community structure to a broader gradient of impact to the coral host, including the ultimate fate of the coral fragments subjected to sublethal increases in temperature.

It is interesting to note that the three $P$. compressa source colonies sampled for this experiment all possessed statistically different microbial communities. The structure of the bacterial communities of two of these colonies were much more closely related to each other than to the third, and, despite changes during the 10-day acclimation phase, this relationship was maintained throughout the duration of the experiment. The maintenance of this relationship throughout the acclimation and experimental periods suggests that the microbial communities either did not randomly diverge upon containment, but rather changed systematically, or that they diverged but the differences did not reach a significant enough magnitude for our methodology to detect. Extension of the duration of the experimental treatment would help to tease apart the interplay between these nonexclusive options.

Throughout the course of this study, several aspects of the experimental aquarium system were identified that had the potential to negatively impact this research by forcing environmental conditions to diverge from the natural environment, and thus would require additional attention in the future. In particular, technical limitations required that the seawater used to fill the experimental aquaria be recirculated within each individual tank. Thus, to limit microbial growth, the seawater initially supplied to the tanks was filtered to remove eukaryotic phytoplankton and treated with UV radiation to decrease the load of viable bacteria. As might be expected, the structure of planktonic bacterial communities within the experimental aquaria appeared to change in an unpredictable fashion over the course of this study, resulting in significant tank differences irrespective of whether they originated from treatment or control groups. While cellular abundance was not monitored, it is probable that the filtration and UV treatments initially decreased the cellular load, but the tank environment provided a hospitable environment for subsequent microbial growth. Ultimately, all tanks exhibited significant changes in planktonic bacterial community structure by the end of the experiment. The source coral colonies sampled in this study were subject to significant, variable flow, and thus experience a constantly refreshed microbial community. While the impact that aquarium-contained planktonic seawater bacterial communities may have on the corals in these aquaria (or their closely associated microbiota) cannot be predicted at the present time it is a potentially confounding factor that can be eliminated by employing a flow-through seawater system with natural seawater as source.

\section{Conclusions}

The primary goal of this study was to begin to explore the relationship between coral-associated bacterial community structure and coral health during an abrupt but sublethal temperature anomaly. More broadly, we sought to relate successional changes in bacterial community structure to gradients of physiological stress in the coral host, before the symptoms of the stress became visually apparent (e.g., bleaching and disease symptoms). While the thermal stress we applied rapidly impacted the photophysiology of the coral host, no changes in the bacterial community structure were detectable with the combination of temperature and duration of treatment used. Data from this study provide new information on the variability of coral-associated bacterial communities amongst colonies of the same species and serve as a guide to improve the design of future experiments aimed at elucidating the role that bacterial communities play in the responses of corals to climate change stressors such as elevated sea surface temperatures and ocean acidification.

\section{Acknowledgments}

The authors would like to thank Chelsea Ale'alani Dudoit for her assistance with field collection and sample processing and Andy Taylor for his assistance with statistical data analysis. This research was funded by a grant/cooperative agreement from the National Oceanic and Atmospheric Administration, Project no. R/CR-12, which is sponsored by the University of Hawaii Sea Grant College Program under Institutional Grant no. NA05OAR4171048 (UNIHI-SEAGRANT-XM-06-03), a research partnership between the Northwestern Hawaiian Island Coral Reef Ecosystem Reserve and the Hawaii Institute of Marine Biology (NMSP MOA 2005-008/66882), and the National Science Foundation (Grant no. OCE-0928806). This is SOEST contribution 8028 and HIMB contribution 1415.

\section{References}

[1] K. D. Lafferty, J. W. Porter, and S. E. Ford, "Are diseases increasing in the ocean?" Annual Review of Ecology, Evolution, and Systematics, vol. 35, pp. 31-54, 2004.

[2] C. Harvell, E. Jordán-Dalhgren, S. Merkel et al., "Coral disease, environmental drivers and the balance between coral and microbial associates," Oceanography, vol. 20, pp. 172-195, 2007.

[3] C. D. Harvell, C. E. Mitchell, J. R. Ward et al., "Climate warming and disease risks for terrestrial and marine biota," Science, vol. 296, no. 5576, pp. 2158-2162, 2002.

[4] J. R. Ward and K. D. Lafferty, "The elusive baseline of marine disease: are diseases in ocean ecosystems increasing?" PLoS Biology, vol. 2, no. 4, pp. 542-547, 2004.

[5] K. G. Kuta and L. L. Richardson, "Ecological aspects of black band disease of corals: relationships between disease incidence and environmental factors," Coral Reefs, vol. 21, no. 4, pp. 393398, 2002.

[6] R. J. Jones, J. Bowyer, O. Hoegh-Guldberg, and L. L. Blackall, "Dynamics of a temperature-related coral disease outbreak," Marine Ecology Progress Series, vol. 281, pp. 63-77, 2004. 
[7] J. M. Cervino, R. L. Hayes, S. W. Polson et al., "Relationship of Vibrio species infection and elevated temperatures to yellow blotch/band disease in Caribbean corals," Applied and Environmental Microbiology, vol. 70, no. 11, pp. 6855-6864, 2004.

[8] Y. Ben-Haim and E. Rosenberg, "A novel Vibrio sp. pathogen of the coral Pocillopora damicornis," Marine Biology, vol. 141, no. 1, pp. 47-55, 2002.

[9] M. E. Brandt and J. W. Mcmanus, "Disease incidence is related to bleaching extent in reef-building corals," Ecology, vol. 90, no. 10, pp. 2859-2867, 2009.

[10] Y. Ben-Haim, E. Banim, A. Kushmaro, Y. Loya, and E. Rosenberg, "Inhibition of photosynthesis and bleaching of zooxanthellae by the coral pathogen Vibrio shiloi," Environmental Microbiology, vol. 1, no. 3, pp. 223-229, 1999.

[11] E. Banin, T. Israely, M. Fine, Y. Loya, and E. Rosenberg, "Role of endosymbiotic zooxanthellae and coral mucus in the adhesion of the coral-bleaching pathogen Vibrio shiloi to its host," FEMS Microbiology Letters, vol. 199, no. 1, pp. 33-37, 2001.

[12] M. P. Lesser, J. C. Bythell, R. D. Gates, R. W. Johnstone, and O. Hoegh-Guldberg, "Are infectious diseases really killing corals? Alternative interpretations of the experimental and ecological data," Journal of Experimental Marine Biology and Ecology, vol. 346, no. 1-2, pp. 36-44, 2007.

[13] T. D. Ainsworth, M. Fine, G. Roff, and O. Hoegh-Guldberg, "Bacteria are not the primary cause of bleaching in the Mediterranean coral Oculina patagonica," ISME Journal, vol. 2, no. 1, pp. 67-73, 2008.

[14] N. Knowlton and F. Rohwer, "Multispecies microbial mutualisms on coral reefs: the host as a habitat," American Naturalist, vol. 162, no. 4, pp. S51-S62, 2003.

[15] F. Rohwer, V. Seguritan, F. Azam, and N. Knowlton, "Diversity and distribution of coral-associated bacteria," Marine Ecology Progress Series, vol. 243, pp. 1-10, 2002.

[16] H. Ducklow and R. Mitchell, "Bacterial populations and adaptations in the mucus layers on living corals," Limnology and Oceanography, vol. 24, no. 4, pp. 715-725, 1979.

[17] M. Wafar, S. Wafar, and J. J. David, "Nitrification in reef corals," Limnology \& Oceanography, vol. 35, no. 3, pp. 725730, 1990.

[18] F. Rohwer, M. Breitbart, J. Jara, F. Azam, and N. Knowlton, "Diversity of bacteria associated with the Caribbean coral Montastraea franksi," Coral Reefs, vol. 20, no. 1, pp. 85-91, 2001.

[19] M. P. Lesser, C. H. Mazel, M. Y. Gorbunov, and P. G. Falkowski, "Discovery of symbiotic nitrogen-fixing cyanobacteria in corals," Science, vol. 305, no. 5686, pp. 997-1000, 2004.

[20] K. B. Ritchie, "Regulation of microbial populations by coral surface mucus and mucus-associated bacteria," Marine Ecology Progress Series, vol. 322, pp. 1-14, 2006.

[21] D. I. Kline, N. M. Kuntz, M. Breitbart, N. Knowlton, and F. Rohwer, "Role of elevated organic carbon levels and microbial activity in coral mortality," Marine Ecology Progress Series, vol. 314, pp. 119-125, 2006.

[22] J. S. Klaus, I. Janse, J. M. Heikoop, R. A. Sanford, and B. W. Fouke, "Coral microbial communities, zooxanthellae and mucus along gradients of seawater depth and coastal pollution," Environmental Microbiology, vol. 9, no. 5, pp. 12911305, 2007.

[23] R. V. Thurber, D. Willner-Hall, B. Rodriguez-Mueller et al., "Metagenomic analysis of stressed coral holobionts," Environmental Microbiology, vol. 11, no. 8, pp. 2148-2163, 2009.
[24] O. Pantos, R. P. Cooney, M. D. A. Le Tissier, M. R. Barer, A. G. O’Donnell, and J. C. Bythell, "The bacterial ecology of a plague-like disease affecting the Caribbean coral Montastrea annularis," Environmental Microbiology, vol. 5, no. 5, pp. 370382, 2003.

[25] R. J. Jones and O. Hoegh-Guldberg, "Effects of cyanide on coral photosynthesis: implications for identifying the cause of coral bleaching and for assessing the environmental effects of cyanide fishing," Marine Ecology Progress Series, vol. 177, pp. 83-91, 1999.

[26] K. Maxwell and G. N. Johnson, "Chlorophyll fluorescence-a practical guide," Journal of Experimental Botany, vol. 51, no. 345, pp. 659-668, 2000.

[27] M. E. Warner, W. K. Fitt, and G. W. Schmidt, "Damage to photosystem II in symbiotic dinoflagellates: a determinant of coral bleaching," Proceedings of the National Academy of Sciences of the United States of America, vol. 96, no. 14, pp. 8007-8012, 1999.

[28] M. T. Suzuki, O. Béjà, L. T. Taylor, and E. F. DeLong, "Phylogenetic analysis of ribosomal RNA operons from uncultivated coastal marine bacterioplankton," Environmental Microbiology, vol. 3, no. 5, pp. 323-331, 2001.

[29] W.-T. Liu, T. L. Marsh, H. Cheng, and L. J. Forney, "Characterization of microbial diversity by determining terminal restriction fragment length polymorphisms of genes encoding 16S rRNA," Applied and Environmental Microbiology, vol. 63, no. 11, pp. 4516-4522, 1997.

[30] C. A. Osborne, G. N. Rees, Y. Bernstein, and P. H. Janssen, "New threshold and confidence estimates for terminal restriction fragment length polymorphism analysis of complex bacterial communities," Applied and Environmental Microbiology, vol. 72, no. 2, pp. 1270-1278, 2006.

[31] K. R. Clarke and R. N. Gorley, PRIMER v6: User Manual/Tutorial, PRIMER-E, Plymouth, UK, 2006.

[32] M. J. Anderson, R. N. Gorely, and K. R. Clarke, PERMANOVA+ for PRIMER: Guide to Software and Statistical Methods, PRIMER-E, Plymouth, UK, 2008.

[33] K. R. Clarke and R. M. Warwick, Change in Marine Communities: An Approach to Statistical Analysis and Interpretation, PRIMER-E, Plymouth, UK, 2nd edition, 2001.

[34] R. N. Shepard, "The analysis of proximities: multidimensional scaling with an unknown distance function. I.," Psychometrika, vol. 27, no. 2, pp. 125-140, 1962.

[35] J. B. Kruskal, "Multidimensional scaling by optimizing goodness of fit to a nonmetric hypothesis," Psychometrika, vol. 29, no. 1, pp. 1-27, 1964.

[36] J. Frias-Lopez, J. S. Klaus, G. T. Bonheyo, and B. W. Fouke, "Bacterial community associated with black band disease in corals," Applied and Environmental Microbiology, vol. 70, no. 10, pp. 5955-5962, 2004.

[37] O. Pantos and J. C. Bythell, "Bacterial community structure associated with white band disease in the elkhorn coral Acropora palmata determined using culture-independent $16 \mathrm{~S}$ rRNA techniques," Diseases of Aquatic Organisms, vol. 69, no. 1, pp. 79-88, 2006.

[38] S. L. Coles and Y. H. Fadlallah, "Reef coral survival and mortality at low temperatures in the Arabian Gulf: new species-specific lower temperature limits," Coral Reefs, vol. 9, no. 4, pp. 231-237, 1991.

[39] P. L. Jokiel and S. L. Coles, "Response of Hawaiian and other Indo-Pacific reef corals to elevated temperature," Coral Reefs, vol. 8, no. 4, pp. 155-162, 1990.

[40] B. E. Brown, "Coral bleaching: causes and consequences," Coral Reefs, vol. 16, no. 1, pp. S129-S138, 1997. 

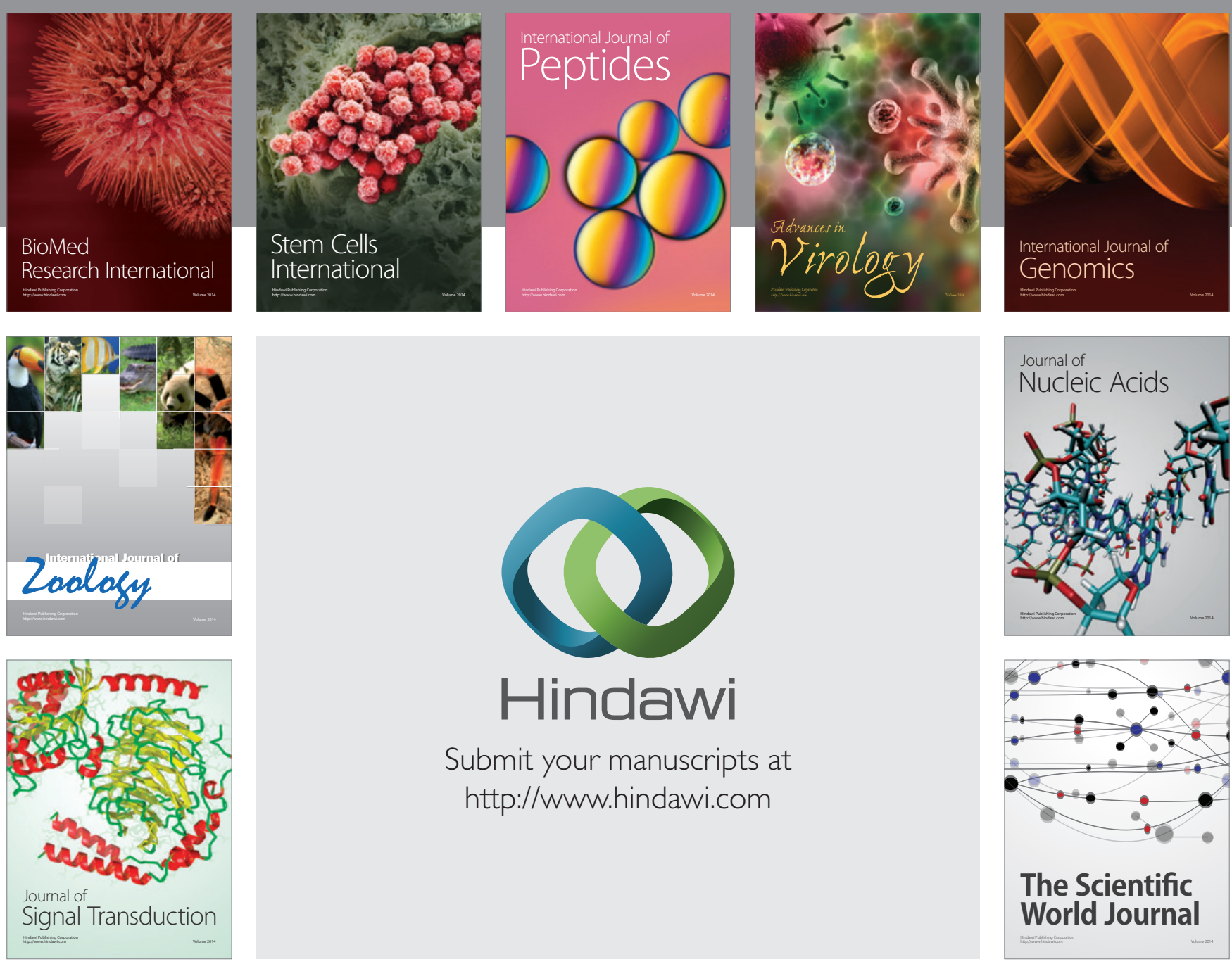

Submit your manuscripts at

http://www.hindawi.com
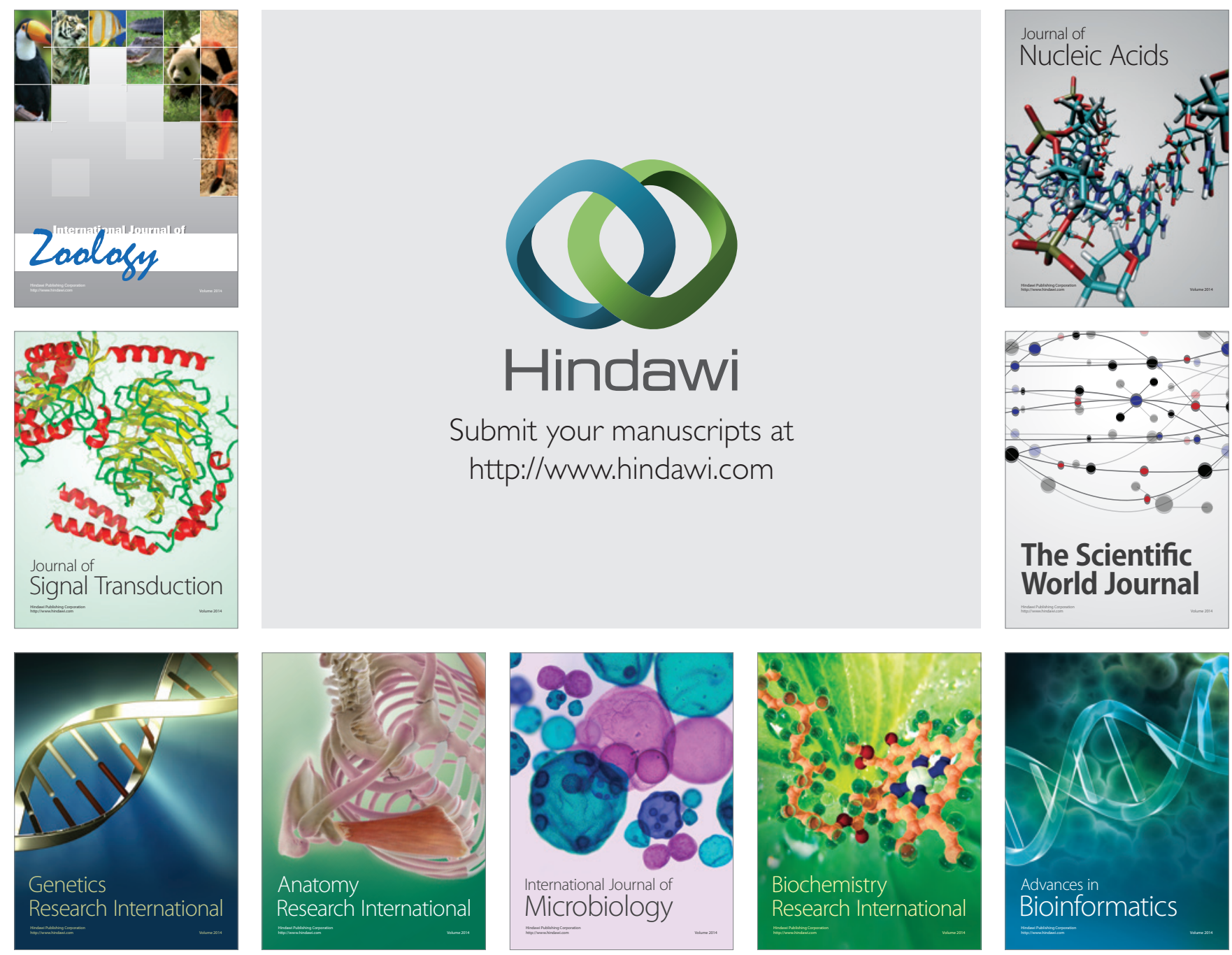

The Scientific World Journal
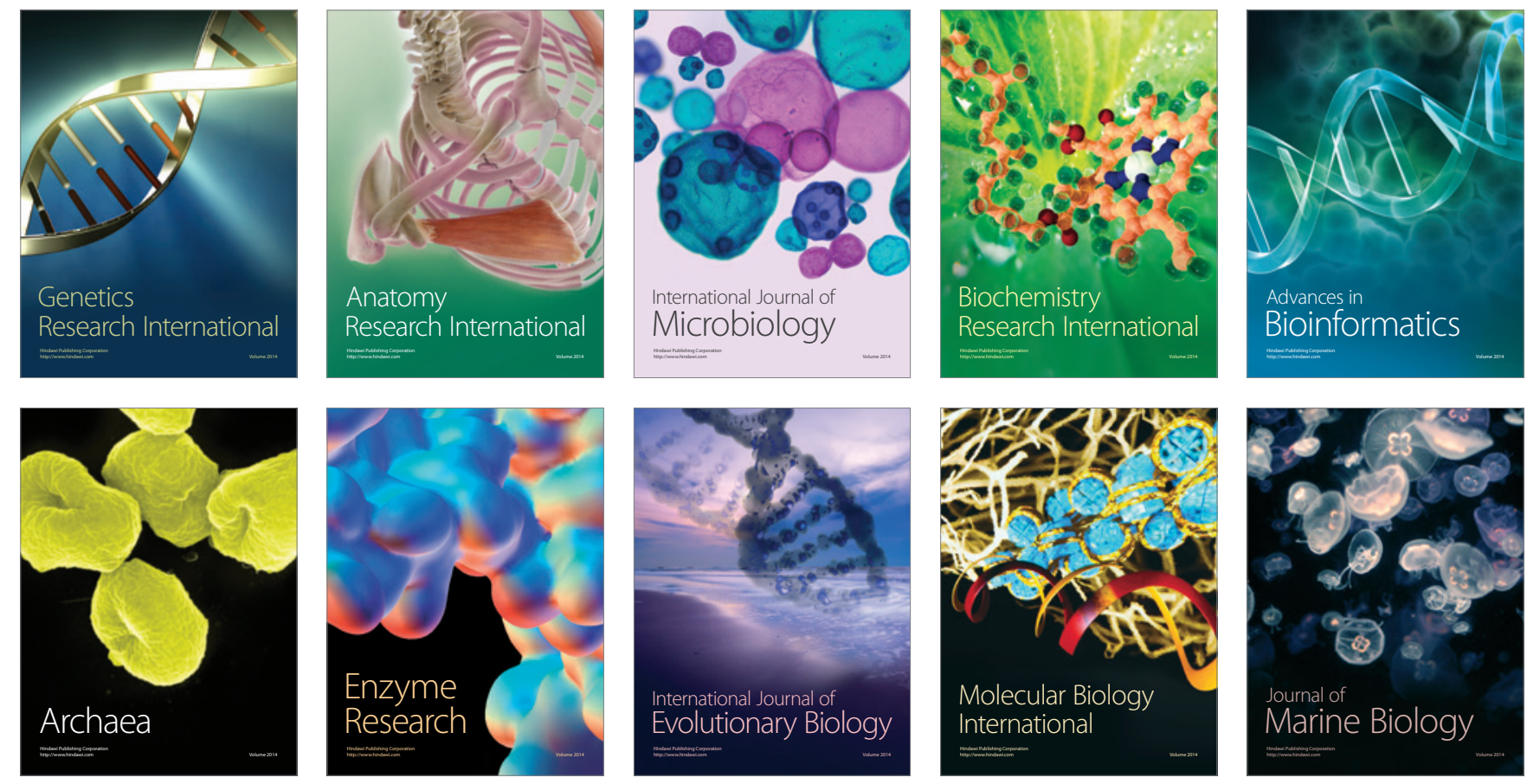